hep-th/0412243

HUTP-04/A034

\title{
Khovanov-Rozansky Homology and Topological Strings
}

\author{
Sergei Gukov ${ }^{1}$, Albert Schwarz ${ }^{2}$, and Cumrun Vafa ${ }^{1}$ \\ 1 Jefferson Physical Laboratory, \\ Harvard University, \\ Cambridge, MA 02138, USA \\ ${ }^{2}$ Department of Mathematics, \\ University of California, \\ Davis, CA 95616, USA
}

\begin{abstract}
We conjecture a relation between the $s l(N)$ knot homology, recently introduced by Khovanov and Rozansky, and the spectrum of BPS states captured by open topological strings. This conjecture leads to new regularities among the $\operatorname{sl}(N)$ knot homology groups and suggests that they can be interpreted directly in topological string theory. We use this approach in various examples to predict the $s l(N)$ knot homology groups for all values of $N$. We verify that our predictions pass some non-trivial checks.
\end{abstract}

Dedicated to the memory of F.A. Berezin.

December 2004 


\section{Introduction and Summary}

During the past twenty years, topological field theories have been the source of the vigorous interaction between theoretical physics and pure mathematics, increasingly fruitful for both fields. One of the famous examples of topological field theories is a Chern-Simons gauge theory [1,2]. Observables in this theory are naturally associated with knots and links. Specifically, given an oriented knot, $K$, and a representation of the gauge group, $R$, one can construct a Wilson loop operator, $W_{R}(K)$, whose expectation value turns out to be a polynomial invariant of $K$, such as the Jones polynomial and its generalizations [2].

Here, we will be mainly interested in the case where $R=\square$ is the fundamental representation of $\operatorname{sl}(N)$. The corresponding quantum invariant

$$
P_{N}(q)=\left\langle W_{\square}(K)\right\rangle
$$

is a one-variable specialization of the HOMFLY polynomial [3]. It can be determined by the $\operatorname{sl}(N)$ skein relation

$$
q^{N} P_{N}\left(L_{+}\right)-q^{-N} P_{N}\left(L_{-}\right)=\left(q^{-1}-q\right) P_{N}\left(L_{0}\right)
$$

and by the normalization

$$
P_{N}(\text { unknot }, q)=[N]=\frac{q^{N}-q^{-N}}{q-q^{-1}}
$$
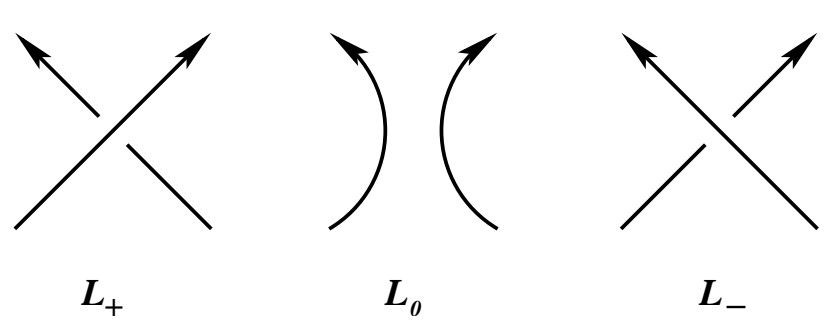

Fig. 1: Link diagrams connected by the skein relation.

As shown in [4], Chern-Simons theory can be embedded in string theory. Later it was shown [5.60.7] using highly non-trivial 'stringy dualities' that this leads to a reformulation of quantum $s l(N)$ knot invariants in terms of topological string amplitudes (GromovWitten invariants). Moreover, it was realized that the polynomial invariants $P_{N}(q)$ can be 
reformulated in terms of integers $N_{\square, Q, s}$ which capture the spectrum of BPS states in the string Hilbert space] [7, [8]:

$$
P_{N}(q)=\frac{1}{q-q^{-1}} \sum_{s, Q \in \mathbb{Z}} N_{\square, Q, s} q^{N Q+s}
$$

It is difficult to give a mathematically rigorous definition of BPS degeneracies. In the simpler case of closed topological strings, there is a similar notion of BPS degeneracies [6]. Attempts to give it a precise mathematical definition [9, 10,11,12,13 led to a deeper understanding of Gromov-Witten invariants, though even there not all questions are answered. In the open string case, even though a mathematically rigorous proof of (1.4) is not available this equation is strongly supported by physical arguments and highly non-trivial checks, in particular, by the fact that considering it as a definition of $N_{\square, Q, s}$ one always obtains integers.

In another line of development, the quantum $s l(N)$ invariant $P_{N}(q)$ was lifted to a homological knot invariant [14,15, 16, 17]. For a given knot $K$ and a fixed value of $N$, the $s l(N)$ homological invariant is a doubly graded cohomology theory, $\mathcal{H}_{N}^{i, j}(K)$, whose Euler characteristic with respect to one of the gradings equals the quantum $\operatorname{sl}(N)$ invariant,

$$
P_{N}(q)=\sum_{i, j \in \mathbb{Z}}(-1)^{i} q^{j} \operatorname{dim} \mathcal{H}_{N}^{i, j}(K)
$$

Following [18], we also introduce the graded Poincaré polynomial,

$$
K h_{N}(q, t):=\sum_{i, j \in \mathbb{Z}} t^{i} q^{j} \operatorname{dim} \mathcal{H}_{N}^{i, j}(K)
$$

Relegating technical details to the following sections, let us summarize some important features of $\mathcal{H}_{N}$ :

i) $\mathcal{H}_{N}$ is a functor (from the category of links and cobordisms to the category of vector spaces [16,18, 19,20]);

ii) $\mathcal{H}_{N}$ is stronger than $P_{N}$, at least in the case $N=2$;

iii) $\mathcal{H}_{N}$ is hard to compute (at present, only the $\operatorname{sl}(2)$ homological invariant has been computed for knots with small number of crossings [18,21]);

iv) $\mathcal{H}_{N}$ cries out for a physical interpretation!

1 In our normalization, the integer invariants $N_{\square, Q, s}$ are non-trivial only for even values of $s$. 
In this paper, we take a modest step towards understanding the physical interpretation of the $\operatorname{sl}(N)$ knot homology by relating it to the BPS spectrum of states in open topological strings. It naturally leads us to new regularities among the homology groups, $\mathcal{H}_{N}^{i, j}(K)$, which we hope may ultimately lead to a better understanding of the $s l(N)$ homological invariant on the deep conceptual level. In particular, we will be interested in the dependence of the homology groups $\mathcal{H}_{N}^{i, j}(K)$ on the rank of $\operatorname{sl}(N)$. By analogy with (1.4), we expect that, at least for sufficiently large values of $N$, the following complex has a very simple structure:

$$
\mathcal{H}_{N}^{\bullet}\{+1\}-\mathcal{H}_{N}^{\bullet}\{-1\}
$$

where $\{n\}$ denotes the shift in $q$-grading up by $n$. Specifically, we propose the following:

Conjecture: For a knot $K$ and sufficiently large日 values of $N$, the graded Poincaré polynomial of the doubly graded complex (1.7) can be expressed in terms of $N$-independent integer invariants $D_{Q, s, r}(K)$,

$$
\left(q-q^{-1}\right) K h_{N}(q, t)=\sum_{Q, s, r \in \mathbb{Z}} D_{Q, s, r} q^{N Q+s} t^{r}
$$

Notice, this conjecture implies that (for sufficiently large $N$ ) the set of values of the grading $i$ in $\mathcal{H}_{N}^{i, j}(K)$ is contained in a set that does not depend on $N$. There is also a generalization of this conjecture for links, see below.

The integer invariants $D_{Q, s, r}$ provide a refinement of the open string BPS degeneracies by decomposition of the states in terms of an additional $U(1)$ charge (grading) $r$ in a sense that

$$
N_{\square, Q, s}=\sum_{r \in \mathbb{Z}}(-1)^{r} D_{Q, s, r} .
$$

This is parallel to the refinement of BPS degeneracies for closed strings found in [22]. Notice, that the conjectured relation (1.8) is manifestly consistent with eqs. (1.4) - (1.6) and the relation (1.9).

\section{Organization of the Paper}

In section 2, we briefly review some of the main statements about integer BPS degeneracies for open strings and the $s l(N)$ knot homology. The more detailed physical setup

2 It turns out, however, that for many simple knots this conjecture works for all $N \geq 2$. 
and the interpretation of the $s l(N)$ knot homology as BPS degeneracies $D_{Q, s, r}$ is discussed in section 3. In section 4, we study explicit examples. In particular, we use (1.8) to predict the $\operatorname{sl}(N)$ homological invariants for certain knots with small number of crossings and verify that our predictions pass some non-trivial checks. In section 5 , we discuss various generalizations of the above conjecture. Finally, in the appendices we present the explicit form of the HOMFLY polynomial and the $\operatorname{sl}(2)$ homological invariant for some knots.

\section{Preliminaries}

\subsection{Conventions}

Here we follow the standard conventions in knot theory, where the unreduced Jones

polynomial has expansion in integer powers of $q, J \in \mathbb{Z}\left[q, q^{-1}\right]$. For example, in these conventions, the quantum $\operatorname{sl}(N)$ invariant of the trivial knot has the form (1.3). These conventions are compatible with the ones used in [14,15, 16, 17, 18], but differ from the conventions used in the physics literature [5, 6, [7, 8, 23, 24]. The two sets of conventions are related by a simple change $q \rightarrow q^{1 / 2}$. We also warn the reader that in some literature our variable $q$ is denoted $q^{-1}$ and, similarly, $t$ is denoted $t^{-1}$.

Throughout, we work over $\mathbb{Q}$. In particular, all cohomology groups are $\mathbb{Q}$-vector spaces, and $\operatorname{dim}\left(\mathcal{H}_{N}^{i, j}\right)$ denotes $\operatorname{dim}_{\mathbb{Q}}\left(\mathcal{H}_{N}^{i, j}\right)$.

\subsection{A Brief Review of the BPS Degenaracies and Knot Invariants}

The generating function of Wilson loop (knot) observables in Chern-Simons theory can be written in terms of the $f$-polynomials [7]:

$$
F_{C S}(V)=\sum_{d=1}^{\infty} \sum_{R} \frac{1}{d} f_{R}\left(q^{d}, \lambda^{n}\right) \operatorname{Tr}_{R} V^{d}
$$

where $\lambda:=q^{N}$ and $N$ is the rank of the gauge group. The polynomials $f_{R}(q, \lambda)$ can be expanded in $q$ and $\lambda[$ [7, 8$]$

$$
\begin{aligned}
f_{R}(q, \lambda) & =\frac{1}{q-q^{-1}} \sum_{s, Q} N_{R, Q, s} \lambda^{Q} q^{s}= \\
& =\sum_{g \geq 0} \sum_{Q} \sum_{R^{\prime}, R^{\prime \prime}} C_{R R^{\prime} R^{\prime \prime}} S_{R^{\prime}}(q) \widehat{N}_{R^{\prime \prime}, g, Q}\left(q^{-1}-q\right)^{2 g-1} \lambda^{Q}
\end{aligned}
$$


In this expression, $R, R^{\prime}$, and $R^{\prime \prime}$ denote representations of the symmetric group, which we can label by Young tableaus with $\ell$ boxes. The coefficients $C_{R R^{\prime} R^{\prime \prime}}$ are the ClebschGordan coefficients of the symmetric group, and the function $S_{R}(q)$ is non-zero only for hook representations of the form

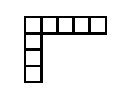

Specifically, if $R$ is a hook representation with $\ell-d$ boxes in the first row, then $S_{R}(q)=$ $(-1)^{d} q^{-\frac{\ell-1}{2}+d}$, and $S_{R}(q)=0$ otherwise. Finally, the numbers $N_{R, Q, s}$ and $\widehat{N}_{R, g, Q}$ encode the BPS spectrum for open topological strings.

The integer BPS degeneracies can be defined in terms of cohomology computations on certain spaces. In particular, for the fundamental represenation, $\widehat{N}_{\square, g, Q}$ can be defined as the Euler characteristics of the cohomology of moduli spaces $⿴ 囗 ⿱ 一 一) \mathcal{M}_{g, Q}$. We refer the reader to the original papers [6,7,8] for the details, and briefly sketch here the results that will be relevant to us in what follows. There is an explicit expression for $\widehat{N}_{\square, g, Q}$ in terms of the cohomology of $\mathcal{M}_{g, Q}$,

$$
\widehat{N}_{\square, g, Q}=\epsilon \chi\left(\mathcal{M}_{g, Q}\right)
$$

wherel $\epsilon= \pm 1$.

In the case we are considering, $R=\square$, the polynomial $f_{\square}(q, \lambda)$ is the unnormalized HOMFLY polynomial. Its value at $\lambda=q^{N}$ gives the $\operatorname{sl}(N)$ polynomial $P_{N}(q)$ :

$$
P_{N}(q)=f_{\square}\left(q, q^{N}\right)
$$

The polynomial $f_{\square}(q, \lambda)$ has a simple representation in terms of $\widehat{N}_{R, g, Q}$ :

$$
f_{\square}(q, \lambda)=\sum_{Q} \sum_{g \geq 0} \widehat{N}_{\square, g, Q}\left(q^{-1}-q\right)^{2 g-1} \lambda^{Q}
$$

Comparing this formula with eq. (2.2), we obtain an expression for the integers $N_{\square, Q, s}$ in terms of $\widehat{N}_{\square, g, Q}$,

$$
N_{\square, Q, s}=-\sum_{g \geq 0}(-1)^{g+s / 2}\left(\begin{array}{c}
2 g \\
g+s / 2
\end{array}\right) \widehat{N}_{\square, g, Q}
$$

Using (2.4), we can further express $N_{\square, Q, s}$ via dimensions of the cohomology groups $H^{k}\left(\mathcal{M}_{g, Q}\right)$,

$$
N_{\square, Q, s}=-\epsilon \sum_{g \geq 0, k}(-1)^{k+g+s / 2}\left(\begin{array}{c}
2 g \\
g+s / 2
\end{array}\right) \operatorname{dim} H^{k}\left(\mathcal{M}_{g, Q}\right)
$$

3 As discussed in section 3 this is the moduli space of holomorphic curves of genus $g$ with one boundary ending on a suitable Lagrangian submanifold.

4 As discussed in [f] the origin of the sign $\epsilon$ is related to the analytic continuation one has to make in order to relate topological string amplitudes with the quantum-group knot invariants. 
Example. The Unknot

For the unknot the corresponding moduli spaces are isolated points, so that

$$
H^{k}\left(\mathcal{M}_{g, Q}\right)= \begin{cases}\mathbb{Q} & \text { if } k=g=0 \text { and } Q= \pm 1 \\ 0 & \text { otherwise }\end{cases}
$$

Hence, from (2.4) we find that the only non-zero invariants are [7]:

$$
\widehat{N}_{\square, 0, Q= \pm 1}=\mp 1
$$

This leads to the usual expression for the HOMFLY polynomial

$$
f_{\square}(\text { unknot })=\frac{\lambda-\lambda^{-1}}{q-q^{-1}}=\frac{q^{N}-q^{-N}}{q-q^{-1}}
$$

Let us consider another example.

Example. The Trefoil Knot

In this case, the non-zero BPS invariants are [8]:

$$
\begin{aligned}
& \widehat{N}_{\square, 0,1}=2, \quad \widehat{N}_{\square, 0,3}=-3, \quad \widehat{N}_{\square, 0,5}=1, \\
& \widehat{N}_{\square, 1,1}=1, \quad \widehat{N}_{\square, 1,3}=-1
\end{aligned}
$$

Substituting this into (2.6), we find

$$
f_{\square}\left(3_{1}\right)=\frac{2 \lambda-3 \lambda^{3}+\lambda^{5}}{q^{-1}-q}+\left(\lambda-\lambda^{3}\right)\left(q^{-1}-q\right)
$$

Notice, that in the rank 2 case, $\lambda=q^{2}$, we recover the usual expression for the unreduced Jones polynomial of the trefoil knot,

$$
J\left(3_{1}\right)=q+q^{3}+q^{5}-q^{9}
$$

\subsection{A Brief Review of the $\operatorname{sl}(N)$ Knot Homology}

In a fascinating work [14], Khovanov introduced a new homological knot invariant, which has the Jones polynomial as its graded Euler characteristic. Subsequently, this work was extended to a categorification of the quantum $\operatorname{sl}(3)$ invariant [16] and, more recently, to a categorification of the quantum $\operatorname{sl}(N)$ invariant [17]. Although these constructions differ in details, the basic idea is to associate a chain complex of graded vector spaces, 
$\mathcal{C}_{N}^{\bullet}(L)$, to a plane diagram of a link $L$ colored by a fundamental representation of $\operatorname{sl}(N)$. The bigraded cohomology groups of this complex,

$$
\mathcal{H}_{N}(L):=H^{*}\left(\mathcal{C}_{N}^{\bullet}\right)
$$

do not depend, up to isomorphism, on the choice of the projection of $L$. It is also convenient to define the graded Poincaré polynomial,

$$
K h_{N}(L):=\sum_{i, j \in \mathbb{Z}} t^{i} q^{j} \operatorname{dim} \mathcal{H}_{N}^{i, j}(L)
$$

and the graded Euler characteristic,

$$
\chi_{q}(L):=\sum_{i, j \in \mathbb{Z}}(-1)^{i} q^{j} \operatorname{dim} \mathcal{H}_{N}^{i, j}(L)
$$

One of the main results in [14,16,17] states that $\chi_{q}(L)$ is equal to the quantum $\operatorname{sl}(N)$ invariant of $L$ :

$$
P_{N}(q)=\chi_{q}(L)=\left.K h_{N}(L)\right|_{t=-1}
$$

Notice, that since the Euler characteristic of the cohomology $\mathcal{H}_{N}^{i, j}(L)$ is the same as the Euler characteristic of the chain complex $\mathcal{C}_{N}^{i, j}(L)$ itself, we can write $(2.17)-(2.18)$ as

$$
P_{N}(q)=\sum_{i, j \in \mathbb{Z}}(-1)^{i} q^{j} \operatorname{dim} \mathcal{C}_{N}^{i, j}(L)
$$

Example. The Trefoil Knot

For the trefoil knot and $N=2$, the Khovanov's invariant $K h_{N=2}(L)$ has the following form:

$$
K h_{N=2}\left(3_{1}\right)=q+q^{3}+q^{5} t^{2}+q^{9} t^{3}
$$

It is easy to see that at $t=-1$ we recover the usual Jones polynomial (2.14) of the trefoil knot.

Now, let us say a few words about the structure of the complex $\mathcal{C}_{N}^{\bullet}(L)$. Let $W=$ $\oplus_{m} W_{m}$ be a graded vector space. Its graded dimension is

$$
\operatorname{dim}_{q} W=\sum_{m} q^{m} \operatorname{dim} W_{m}
$$


In the spirit of the derived categories, we will be considering chain complexes of these graded vector spaces, e.g.

$$
\mathcal{C}^{\bullet}: \quad \ldots \stackrel{d^{r-1}}{\longrightarrow} \mathcal{C}^{r} \stackrel{d^{r}}{\longrightarrow} \mathcal{C}^{r+1} \stackrel{d^{r+1}}{\longrightarrow} \mathcal{C}^{r+2} \stackrel{d^{r+2}}{\longrightarrow} \ldots
$$

where $r$ is the "height" of the graded vector space $\mathcal{C}^{r}$.

Let us also introduce two translation functors: the degree shift

$$
W\{l\}_{m}:=W_{m-l}
$$

and the height shift

$$
\mathcal{C}[s]^{r}:=\mathcal{C}^{r-s}
$$

In other words, $\mathcal{C}^{\bullet}[s]$ denotes the complex $\mathcal{C}^{\bullet}$ shifted $s$ places to the right. Also, note that (2.23) implies

$$
\operatorname{dim}_{q} W\{l\}=q^{l} \operatorname{dim}_{q} W
$$
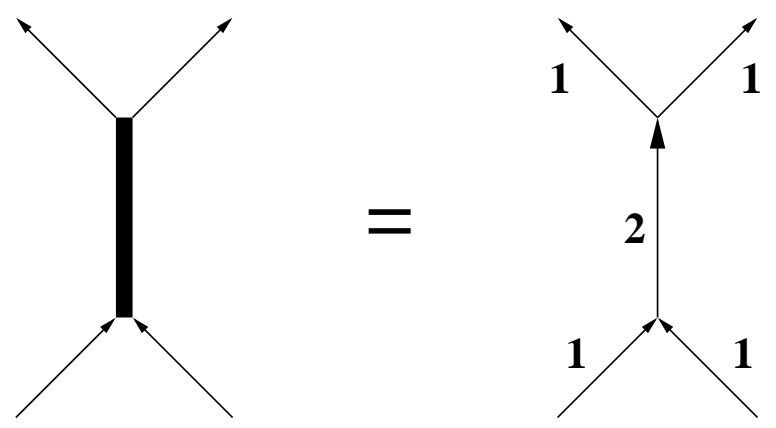

Fig. 2: A planar trivalent graph $\Gamma$ near a "wide edge" and its representation in the Murakami-Ohtsuki-Yamada terminology [25], where each oriented edge is labelled by a fundamental weight of $\operatorname{sl}(N)$.

For each value of $N$, there is a combinatorial algorithm to construct the $\mathbb{Z} \oplus \mathbb{Z}$-graded chain complex $\mathcal{C}_{N}^{\bullet}(L)$ from a plane diagram $D$ of the link $L$. Namely, using a suitable skein relation one takes all possible resolutions of the link diagram $D$,

$$
\mathcal{C}_{N}^{\bullet}(L)=\bigoplus_{\Gamma} \mathcal{C}_{N}^{\bullet}(\Gamma)
$$

where each resolution $\Gamma$ is a planar trivalent graph, as in fig. 2. For example, in the $\operatorname{sl}(2)$ case, each $\Gamma$ is a collection of plane cycles, and a simple algorithm for constructing $\mathcal{C}_{2}^{\bullet}(L)$ was proposed by Khovanov in the original paper [14]; essentially, one has

$$
\mathcal{C}_{2}^{\bullet}(\Gamma) \cong V^{\otimes \#(\text { cycles })}
$$


Here, $V$ is a cohomology ring of a 2 -sphere, $V \cong H^{*}\left(\mathbf{S}^{2}\right)$, so that $\operatorname{dim}_{q}(V)=q+q^{-1}$. Similarly, in the $s l(3)$ case, one can systematically construct $\mathcal{C}_{3}^{\bullet}(L)$ using web cobordisms ("foams") [16].

A general algorithm, which in principle for arbitrary $N$, was proposed recently by Khovanov and Rozansky [17]. In this approach, $\mathcal{C}_{N}^{\bullet}(L)$ is constructed as a $\mathbb{Z} \oplus \mathbb{Z} \oplus \mathbb{Z}_{2}$-graded complex of $\mathbb{Q}$-vector spaces. It turns out that the cohomology groups of this complex are non-trivial only for one value of the $\mathbb{Z}_{2}$ grading, so that $H^{*}\left(\mathcal{C}_{N}^{\bullet}\right)$ is $\mathbb{Z} \oplus \mathbb{Z}$-graded as in (2.15). Basically, a 2-periodic complex $\mathcal{C}_{N}^{\bullet}(L)$ is a tensor product of matrix factorizations:

$$
M^{0} \stackrel{d^{0}}{\longrightarrow} M^{1} \stackrel{d^{1}}{\longrightarrow} M^{0}
$$

We remind that a matrix factorization of a homogeneous potential $W\left(x_{i}\right)$ is a collection of two free modules $M^{0}, M^{1}$ over the ring of polynomials in $x_{i}$, and two maps $d^{0}: M^{0} \rightarrow$ $M^{1}$ and $d^{1}: M^{1} \rightarrow M^{0}$, such that

$$
d^{0} d^{1}=W \cdot \mathrm{Id} \quad, \quad d^{1} d^{0}=W \cdot \mathrm{Id}
$$

Choosing a basis in $M^{0}, M^{1}$ we can think of $d^{0}$ and $d^{1}$ as matrices with polynomial entries. One can also combine $d^{0}$ and $d^{1}$ into an odd matrix of the form

$$
\mathcal{Q}=\left(\begin{array}{cc}
0 & d^{1} \\
d^{0} & 0
\end{array}\right)
$$

so that (2.29) can be written in a compact form

$$
\mathcal{Q}^{2}=W \cdot \operatorname{Id}
$$

In other words, $\mathcal{Q}$ can be regarded as an odd endomorphism (also known as "twisted differential") acting on a free $\mathbb{Z}_{2}$-graded module $M=M^{0} \oplus M^{1}$ over $R=\mathbb{Q}\left[x_{i}\right]$. When, following Khovanov and Rozansky [17], one takes a tensor product over matrix factorizations of the form (2.28), the $W$ 's cancel, so that $\mathcal{Q}$ becomes an ordinary differential, $\mathcal{Q}^{2}=0$, and the result becomes a complex,

$$
\mathcal{C}_{N}^{\bullet}(L)=\otimes_{i} M_{i}
$$

5 In practice, the previous two constructions remain more suitable for explicit calculations of the $\operatorname{sl}(2)$ and $\operatorname{sl}(3)$ knot homology. 
A factorization $M$ is called trivial (or contractible) if it has the form

$$
R \stackrel{1}{\longrightarrow} R \stackrel{W}{\longrightarrow} R
$$

Dividing the category of matrix factorizations (2.28) by the equivalence relation

$$
M \sim M \oplus M_{\text {trivial }}
$$

we get a "derived" category of factorizations of $W$, denoted by $\mathrm{hmf}_{W}$ in [17], whose isomorphisms are morphisms which induce isomorphisms in cohomology. Furthermore, this category has a triangulated structure, which plays an important role in the notion of D-brane stability, see [26] for a recent review. In fact, the category $\mathrm{hmf}_{W}$ is a simple example of a "D-brane category", whose objects are D-branes in $\mathcal{N}=2$ Landau-Ginzburg model with a superpotential $W$ 22, 28,29,30. In particular, with each "twisted complex," $M_{i} \in \operatorname{hmf}_{W_{i}}$, we can associate a Landau-Ginzburg model and a D-brane in it. The tensor product of these Landau-Ginzburg models is a two-dimensional $\mathcal{N}=2$ theory, $c f$. [31,32,33,34:

$$
\otimes_{i} \mathrm{LG}_{W_{i}}
$$

We refer the reader to [14, 16, 17, 18] for further details on the construction of the $\operatorname{sl}(N)$ knot homology.

\section{Physical Interpretation of the $s l(N)$ Knot Cohomology}

In this section we start by briefly reviewing the relations between BPS states and topological strings. We then discuss how Chern-Simons theory on $M$ is embedded in open topological strings on $T^{*} M$. We also review how in the case of $M=\mathbf{S}^{3}$ 'large N' stringy dualities relate it to closed topological string on a deformed geometry (the small resolution of the nodal singularity). Also reviewed is how the HOMFLY polynomial invariants can be formulated in this context. We then go on to define a refined version of the BPS degeneracies which leads to a refinement of open topological string amplitudes. We then propose a relation between this refined BPS degeneracies to the $\operatorname{sl}(N)$ knot homology which is a categorification of quantum $s l(N)$ polynomial invariants.

\footnotetext{
${ }^{6}$ For a more detailed review on these topics the reader can consult [35].
} 


\subsection{Topological Strings and BPS Degeneracies}

Closed topological string, which encodes Gromov-Witten invariants, deals with holomorphic maps from closed Riemann surfaces of arbitrary genus to Calabi-Yau threefolds $X$. The partition function $Z$ is a function of the Kähler moduli of Calabi-Yau threefold, which we denote by $T$ and of the string coupling constant $g_{s}$. The dependence on $T$ arises by weighing each map by $\exp (-A)$ where $A$ is the area of the curve. The $g_{s}$ dependence enters by weighing a genus $g$ Riemann surface by the factor $g_{s}^{2 g-2}$. It has been argued in [6] that $Z$ can be rewritten in terms of integers $n_{Q}^{k}$ as

$$
Z\left(T, g_{s}\right)=\prod_{k, Q}\left[\prod_{n=1}^{\infty}\left(1-q^{2 n+2 k} e^{-\langle Q, T\rangle}\right)^{n}\right]^{n_{Q}^{k}}
$$

where $q=e^{-g_{s} / 2}$ and $Q$ denotes an element of $H_{2}(X, \mathbb{Z})$. The integers $n_{Q}^{k}$ denote the degeneracy of BPS states. More precisely these correspond to embedded holomorphic curves in the class $Q$ and the index $k$ is related to the genus of the curve. Physically these degeneracies are computed by studying the moduli spaces of D-branes in the class $Q$. Note that the moduli space includes the choice of a flat connection on the D-brane in addition to the moduli associated to moving the D-brane inside $X$. Let us consider an idealized situation (the more general case was discussed in [11]; see also [9, 10, 12, 13]): Suppose that as we vary the curve inside $X$ its genus does not change (and assume there are no monodromies of the cycles of the Riemann surface). In this case the moduli space naturally splits into the moduli space of the flat connection, which is a $2 g$ dimensional torus $T^{2 g}$, and that of the geometric moduli $\mathcal{M}_{\text {Geom. }}^{Q}$ :

$$
\mathcal{M}^{Q}=T^{2 g} \times \mathcal{M}_{\text {Geom }} .
$$

In this simple situation one finds that

$$
\sum_{k} n_{Q}^{k} q^{k}=\chi\left(\mathcal{M}_{\text {Geom. }}\right) \cdot\left(q-q^{-1}\right)^{2 g}
$$

Here the factor $\left(q-q^{-1}\right)^{2 g}$ corresponds to the Poincaré polynomial of $T^{2 g}$. The reader may wonder why the Poincaré polynomial for the full space $\mathcal{M}^{Q}$ does not enter the topological string amplitudes, and only the Euler characteristic of the geometric moduli enters? The reason is that as one changes the complex moduli of Calabi-Yau $X$, it turns out that $\mathcal{M}_{\text {Geom. }}$. changes topology (and even dimension!), but its Euler characteristic does not change. So only $\chi$ is an invariant of the Calabi-Yau $X$. 
However, there are cases where $X$ is rigid and has no complex moduli. In such cases one could have obtained a more refined invariant by introducing a new parameter $t$ which captures the Poincaré polynomial $P_{\mathcal{M}_{\text {Geom }}}(t)$ of the geometric moduli. In the physical context this corresponds to an addition 'charge' that we can measure and refine the degeneracies to $n_{Q}^{k, k^{\prime}}$. This extra charge appears as follows: In compactifications on M-theory on Calabi-Yau 3-folds we end up with a four dimensional space $\mathbb{R}^{4}$. The rotation symmetry group is thus $S O(4)=S U(2)_{L} \times S U(2)_{R}$. The charge measured by powers of $q$ (together with $(-1)^{F}$ ) corresponds to $U(1)_{L} \in S U(2)_{L}$. We could also measure the charge $U(1)_{R} \in S U(2)_{R}$. So we would have considered

$$
\sum_{k, k^{\prime}} n_{Q}^{k, k^{\prime}} q^{2 k} t^{k^{\prime}}=\left(q-q^{-1}\right)^{2 g} P_{\mathcal{M}_{G e o m}}(t)
$$

In fact in view of the application to the knots it is natural to redefine the basis of the $U(1)_{L} \times U(1)_{R}$ charges. This is because in that case we have to introduce Lagrangain D-branes which fill $\mathbb{R}^{2} \subset \mathbb{R}^{4}$. We still have a $U(1)^{\otimes 2}$ symmetry. However one of the $U(1)$ 's corresponds to the physical spin in the subspace $\mathbb{R}^{2}$ and is given by the diagonal in $U(1)_{L} \times U(1)_{R}$. We will thus redefind

$$
q \rightarrow i q t^{\frac{1}{2}}
$$

and write

$$
P_{Q}(q, t)=\sum_{k, k^{\prime}} n_{Q}^{k, k^{\prime}}\left(q^{2} t\right)^{k}\left(t / q^{2}\right)^{k^{\prime}}=(-1)^{g}\left(q t^{\frac{1}{2}}+q^{-1} t^{-\frac{1}{2}}\right)^{2 g} P_{\mathcal{M}_{\text {Geom }}}(t)
$$

This structure of course presupposes that the moduli space $\mathcal{M}^{Q}$ has a product structure. This is seldom the case. One case where this happens naturally is when the geometric moduli space is a point. One may think that in the more general case the splitting of the Poincaré polynomial to two variable polynomials may not exist. However physical reasoning, i.e. the existence of two $U(1)$ charges that one can measure, guarantees that this must be possible even if the moduli space does not split. Moreover if $X$ is rigid it is an invariant for the Calabi-Yau. Examples of this have already been computed and checked against physical computations in [22], see also [10,36] for a mathematical discussion. This

7 The choice of $i=\sqrt{-1}$ and also the factor of $1 / 2$ in the exponent of $t$ in the formula is for convenience of comparison with the conventions used in the knot theory literature. 
means that $P_{Q}(q, t)$ always exists but it does not in general take the simple form given above as a product of two prefactors.

One can also consider open topological strings. To do this we consider Lagrangian subspaces $\mathcal{L}_{i}$ of $X$ with some multiplicity $N_{i}$ associated to each. We then consider holomorphic maps from Riemann surfaces with arbitrary boundaries such that the boundaries all lie on some Lagrangian brane $\mathcal{L}_{i}$. Each such configurations will receive a factor $N_{i}$; or if the boundary circle wraps over the cycle $\mathbf{S}^{1} r$ times, with holonomy factor $U_{i}$, we pick up a factor of $\operatorname{tr} U_{i}^{r}$. The open topological string amptitudes can again be recast in terms of the BPS degeneracies, D2 branes embedded in $X$ which can have a boundary on the Lagrangian submanifold. The degeneracies will also have a label of Representation of the brane, encoding different ways the D2 branes end on the Lagrangian submanifold. The story in this case is somewhat more complicated that in the closed string case [7],8] . However just as in the closed string case one expects the counting of these degeneracies to depend on choice of charge $Q$ in the relative homology $H_{2}\left(X, \mathcal{L}_{i}\right)$, the spin $s$ and in addition on the representation label $R, N_{R, Q, s}$, which is related to cohomology computations on the moduli space of D2 branes with boundaries (again including the flat connection). The open topological string amplitudes can be written in terms of $N_{R, Q, s}$ as discussed in (2.1).

What we would like to ask now is whether we can define a more refined invariant even in this case, in other words, are there more physical charges we can measure? Let us recall how the open string BPS charges get embedded in the closed string charges [7, 8]: As noted above we have Lagrangian D-branes filling $\mathbb{R}^{2} \subset \mathbb{R}^{4}$ and thus in the open string context we still have both $U(1)$ symmetries. Let us denote these two charges by $(s, r)$, where $s$ denotes the spin in $\mathbb{R}^{2}$. Thus we expect that any degeneracies can also be further refined according to these two charges, in particular we would get new degeneracies $\widetilde{D}_{R, Q, s, r}$ which have the property that

$$
N_{R, Q, s}=\sum_{r}(-1)^{r} \widetilde{D}_{R, Q, s, r}
$$

One could ask if the integers $\widetilde{D}_{R, Q, s, r}$ are invariant, i.e. do they depend on moduli of Calabi-Yau or moduli of the brane? It is easy to see, just as in the closed string case, that they could depend on the complex structure moduli of the Calabi-Yau. But they cannot depend on the Kähler moduli of the Calabi-Yau, or the moduli of the brane. The latter statement follows from the fact that the BPS states are in the $(c, c)$ multiplets whereas the Kähler moduli of Calabi-Yau or the brane moduli are in the $(a, c)$ multiplets and so it 
cannot give mass to the $(c, c)$ multiplets. In particular if we are dealing with a rigid CalabiYau we would find the more refined invariants $\widetilde{D}_{R, Q, s, r}$, similar to what we discussed for the closed string BPS states.

In this paper for simplicity we will mainly concentrate on the case where $R=\square$, i.e., $N_{\square, Q, s}$ and the corresponding refinement will be denoted by $\widetilde{D}_{Q, s, r}$. For the representation $R=\square$ the only relevant BPS brane are Riemann surfaces with one boundary on the Lagrangian submanifold. Suppose we have an isolated Riemann surface of genus $g$ with one boundary. Then, just as we discussed in the closed string case we would find the refined Poincaré polynomial:

$$
\sum_{s, r} \widetilde{D}_{Q, s, r} q^{s} t^{r}=(-1)^{g}\left(q t^{\frac{1}{2}}+q^{-1} t^{-\frac{1}{2}}\right)^{2 g}
$$

\subsection{Chern-Simons Theory and Open Topological Strings}

It was shown in [4] that $U(N)$ Chern-Simons theory on a three manifold $M$ can be reformulated in terms of open topological string on the Calabi-Yau manifold $X=T^{*} M$, where we consider $N$ D-branes wrapping the Lagrangian cycle $M$. What this means is that we consider Riemann surfaces with boundaries and 'count' holomorphic maps from the Riemann surface onto $T^{*} M$ with the condition that the boundary ends on $M$, and that each boundary gets a factor of $N$ corresponding to which brane it ends on. Moreover the Chern-Simons coupling $2 \pi i /(k+N)$ gets identified with topological string coupling constant $g_{s}$. In fact there are no honest holomorphic maps from Riemann surfaces to $T^{*} M$ however we end up with degenerate maps which approach a holomorphic map. These degenerate maps correspond to ribbon graphs on $M$ and in fact reproduce the Feynman graphs of the Chern-Simons perturbation theory.

\subsection{Open Topological String on $\mathbf{S}^{3}$ and a Large $N$ Duality}

It was shown in [5] that for the case of $M=\mathbf{S}^{3}$, with $N$ branes wrapping $\mathbf{S}^{3}$ there is an equivalent description of the open topological strings in terms of a closed topological string. The corresponding Calabi-Yau is obtained by a geometric transition: $\mathbf{S}^{3}$ shrinks to a point, leading to conifold which can be smoothed out by a small blow up leading to a Calabi-Yau geometry which is a total space of the bundle $\mathcal{O}(-1) \oplus \mathcal{O}(-1)$ over $\mathbf{P}^{1}$. Moreover the Kähler moduli of $\mathbf{P}^{1}$ (i.e. its area) is $T=N g_{s}=2 \pi i N / k+N$. In other words

$$
\exp (-T / 2)=\lambda=q^{N}
$$

This therefore gives a relation between Chern-Simons theory on $\mathbf{S}^{3}$ and closed topological strings on the resolved conifold. 


\subsection{Introducing the Knots}

One can extend the above relation between Chern-Simons theory on $\mathbf{S}^{3}$ and topological strings on resolved conifold, to include knot observables [7]: Consider a knot $K$ in $\mathbf{S}^{3}$. $K$ defines a non-compact Lagrangian submanifold $\mathcal{L}_{K}$ in $T^{*} \mathbf{S}^{3}$ given by the conormal bundle. If we wrap $M$ D-branes on $\mathcal{L}_{K}$ we also get a Chern-Simons theory on it. We have two Wilson loop observables $U, V$ on $\mathbf{S}^{3}$ and $\mathcal{L}_{K}$. Viewing $V$ as fixed, wrapping branes on $\mathcal{L}_{K}$ deforms the Chern-Simons theory on $\mathbf{S}^{3}$ and gives a new partition function:

$$
Z(V, N, k)=\sum_{R} \operatorname{tr}_{R} V\left\langle\operatorname{tr}_{R} U\right\rangle=\sum_{R}\left(\operatorname{tr}_{R} V\right) W_{R}(K)
$$

The large $N$ duality relates this to computation of topological strings on the resolved conifold where the branes wrapping $\mathbf{S}^{3}$ have disappeared, but the branes wrapping $\mathcal{L}_{K}$ continue to be there. The fact that there should be such a Lagrangian $\mathcal{L}_{K}$ even after the transition has been shown in [37]. This means that we can rewrite $Z(V, N, k)$ in terms of open topological strings in this new geometry. The $N$ and $k$ dependence capture the Kähler moduli dependence and the string coupling constant, and the $V$ dependence captures how the Riemann surface ends on $\mathcal{L}_{K}$ (if a boundary wraps around the $\mathbf{S}^{1} \subset \mathcal{L}_{K} r$ times it leads to a factor $\operatorname{tr} V^{r}$ ). In this case the Calabi-Yau is rigid and so we can define the refined

invariants $\widetilde{D}_{R, Q, s, r}$ associated to each knot $K$. For the case of $R=\square$ it is natural to expect this is related to $\operatorname{sl}(N)$ knot homology. Let us try to see if we can make this connection more precise.

From the definition of open topological string amplitude and its relation to quantum $s l(N)$ knot invariant (1.4) it follows that

$$
P_{N}(q)=\frac{1}{q-q^{-1}} \sum_{Q, s, r \in \mathbb{Z}}(-1)^{r} q^{N Q+s} \widetilde{D}_{Q, s, r}
$$

where we also used (3.2). It is tempting in the above formula to replace $(-1)$ by a new variable $t$ and write the generating function of the refined BPS degeneracies $\widetilde{D}_{Q, s, r}$ in the form

$$
\frac{1}{q-q^{-1}} \sum_{Q, s, r \in \mathbb{Z}} \widetilde{D}_{Q, s, r} q^{N Q+s} t^{r}
$$

We expect, that there is a similar expression for the graded Poincaré polynomial of the $s l(N)$ homological invariant in terms of some integers $D_{Q, s, r}, c f$. (1.8):

$$
K h_{N}(q, t)=\frac{1}{q-q^{-1}} \sum_{Q, s, r \in \mathbb{Z}} D_{Q, s, r} q^{N Q+s} t^{r}
$$


where $D_{Q, s, r}$ obey

$$
N_{\square, Q, s}=\sum_{r \in \mathbb{Z}}(-1)^{r} D_{Q, s, r}
$$

The above statement, if true, would give a highly non-trivial prediction for the $N$ dependence of the homological $\operatorname{sl}(N)$ invariants. In fact, we find this dependence is satisfied in all the examples we have checked, as will be discussed in the next section.

Although we do not claim that the generating functions (3.5) and (3.6) need to be equal, we expect a simple relation between the refined BPS invariants, $D_{Q, s, r}$, and their physical counterparts, $\widetilde{D}_{Q, s, r}$. Indeed, in the examples considered below, one can recognize contributions of genus- $g$ curves of the form

$$
\sum_{s, r} D_{Q, s, r} q^{s} t^{r}=(-1)^{g}\left(q t^{\frac{1}{2}}+q^{-1} t^{-\frac{1}{2}}\right)^{2 g} t^{\alpha_{K} Q}
$$

where $\alpha_{K}$ is some simple invariant of the knot $K$. Comparing this expression with (3.1) and (3.3), one can interpret such terms either as contributions of isolated curves, in which case $P_{\mathcal{M}_{\text {Geom }}}(t)=1$, or as contributions 8 of genus- $g$ curves with $P_{\mathcal{M}_{\text {Geom }}}(t)=t^{\alpha_{K} Q}$. In the first case, we expect the extra factor of $t^{\alpha_{K} Q}$ to come from the change of basis for $(Q, s, r)$ in the relation between $D_{Q, s, r}$ and $\widetilde{D}_{Q, s, r}$ :

$$
D_{Q, s, r}=\widetilde{D}_{Q, s, r-\alpha_{K} Q}
$$

This change of basis would be very natural in a relation between two graded homology theories. Notice, it does not affect the $N$-dependence of (3.5) - 3.6) which is true even without such a shift. It would be important to understand this change of basis more deeply. It is tempting to speculate that this is related to replacing the $s l(N)$ knot homology with a $g l(N)$ version, as the corresponding Chern-Simons theory related to topological strings is the $U(N)$ version and not the $S U(N)$ version.

On the other hand, if we interpret (3.8) as a contribution of genus- $g$ curve with $P_{\mathcal{M}_{\text {Geom }}}(t)=t^{\alpha_{K} Q}$, there is no need for the change of basis $r \rightarrow r-\alpha_{K} Q$, and we can simply identify $D_{Q, s, r}=\widetilde{D}_{Q, s, r}$.

\footnotetext{
8 Such contributions can arise if there is a non-trivial monodromy.
} 


\section{Examples}

\subsection{The Unknot}

The conjecture (1.8) automatically holds for the unknot, the only knot for which all the $\operatorname{sl}(N)$ knot homology groups are known at present.

Indeed, for the unknot, the $s l(N)$ knot cohomology coincides with its graded Euler characteristic (1.3) (since all the non-trivial groups $\mathcal{H}_{N}^{i, j}$ (unknot) are only in degree $i=0$ ). Therefore, the graded Poincaré polynomial manifestly obeys the relation (1.8),

$$
K h_{N}(q, t)=\frac{1}{q-q^{-1}} \sum_{Q, s, r} D_{Q, s, r} q^{N Q+s} t^{r}
$$

with non-zero invariants

$$
D_{-1,0,0}=-1, \quad D_{1,0,0}=1
$$

Notice, that, in the case of the unknot, the moduli spaces $\mathcal{M}_{g, Q}$ are isolated points when $Q=1$ and $Q=-1$, cf. (2.9). These are precisely the values of $Q$ for which we find non-trivial invariants (4.1). Moreover, for each value of $Q$, the invariants (4.1) have the structure (3.3) consistent with a contribution of an isolated curve with $g=0$.

Let us consider the explicit form of the $\operatorname{sl}(N)$ cohomology groups for the unknot. For example, for $N=4$ we have

$$
\begin{array}{llllllllllllll}
\mathcal{H}_{N=4}^{i, j}: & 0 & 0 & 0 & 0 & 0 & 0 & 0 & 0 & 0 & 0 & 0 & 0 & 0 \\
& 0 & 0 & 0 & 0 & 0 & 0 & 0 & 0 & 0 & 0 & 0 & 0 & 0 \\
& 0 & 0 & 0 & \mathbb{Q} & 0 & \mathbb{Q} & 0 & \mathbb{Q} & 0 & \mathbb{Q} & 0 & 0 & 0
\end{array}
$$

where the vertical direction represents the index $i$, while the horizontal direction represents the grading $j$. In general, the complex $\mathcal{H}_{N}^{\bullet}$ (unknot) has $N$ non-trivial elements. On the other hand, the complex (1.7) obtained from (4.2) has only two non-trivial elements,

$$
\begin{array}{ccccccccccccc}
0 & 0 & 0 & 0 & 0 & 0 & 0 & 0 & 0 & 0 & 0 & 0 & 0 \\
0 & 0 & 0 & 0 & 0 & 0 & 0 & 0 & 0 & 0 & 0 & 0 & 0 \\
0 & 0 & \ominus \mathbb{Q} & 0 & 0 & 0 & 0 & 0 & 0 & 0 & \mathbb{Q} & 0 & 0
\end{array}
$$

where we used the equivalence $\mathbb{Q} \ominus \mathbb{Q} \cong 0$. 


\subsection{The Trefoil Knot}

In this case, the complex (1.7) has six non-trivial elements9. Similarly, there are only six non-zero BPS invariants $N_{\square, Q, s}$ for the following values of $(Q, s)$ :

$$
(1,-2), \quad(3,-2), \quad(1,2), \quad(3,0), \quad(3,2), \quad(5,0)
$$

It is natural to expect that each of the non-zero BPS invariants comes from a single cohomology group which, in turn, corresponds to a particular element in (1.7) with the same value of $(Q, s)$ :

$$
N_{\square, Q, s}=(-1)^{r} D_{Q, s, r}
$$

Notice, there is no summation over $r$ in this formula. There is a unique choice of such $D_{Q, s, r}$ consistent with the $s l(2)$ homological invariant (2.20):

$$
\begin{aligned}
& D_{3,-2,0}=1, \quad D_{3,0,2}=1, \quad D_{5,0,3}=1, \\
& D_{1,-2,0}=-1, \quad D_{1,2,2}=-1, \quad D_{3,2,3}=-1
\end{aligned}
$$

Given these numbers, one can use (1.8) to compute all $s l(N)$ cohomology groups for the trefoil knot. For example, substituting (4.7) into (1.8) we find the following homological sl(3) invariant:

$$
K h_{N=3}\left(3_{1}\right)=q^{2}+q^{4}+q^{6}+q^{6} t^{2}+q^{8} t^{2}+q^{12} t^{3}+q^{14} t^{3}
$$

Remarkably, this result is in perfect agreement with the one obtained using the technique of foams 10 [16]. For the $s l(4)$ and $s l(5)$ homology, we predict

$$
K h_{N=4}\left(3_{1}\right)=q^{3}+q^{5}+q^{7}+q^{9}+q^{7} t^{2}+q^{9} t^{2}+q^{11} t^{2}+q^{15} t^{3}+q^{17} t^{3}+q^{19} t^{3}
$$

9 For example, for $N=2$ it has the form

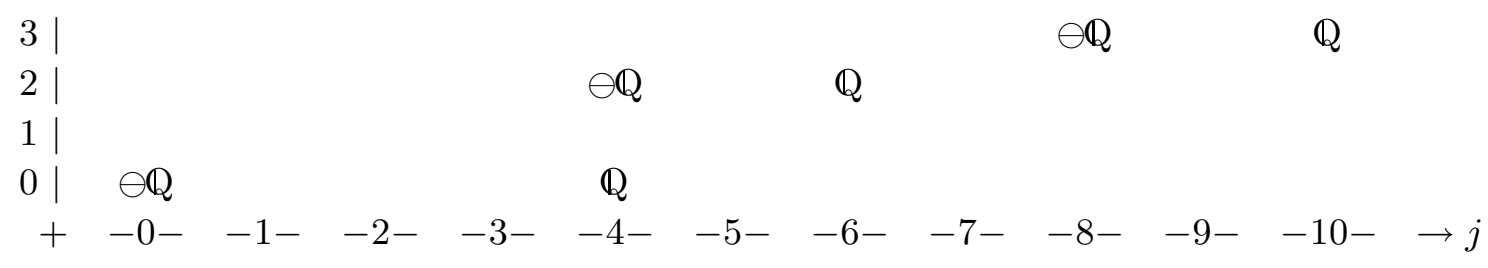

where the vertical (resp. horizontal) direction represents index $i$ (resp. index $j$ ) and we used $\mathbb{Q} \ominus \mathbb{Q} \cong 0$.

10 We thank M. Khovanov for explanations and very helpful discussions on the "computational shortcuts" used in these computations. 
$K h_{N=5}\left(3_{1}\right)=q^{4}+q^{6}+q^{8}+q^{10}+q^{12}+q^{8} t^{2}+q^{10} t^{2}+q^{12} t^{2}+q^{14} t^{2}+q^{18} t^{3}+q^{20} t^{3}+q^{22} t^{3}+q^{24} t^{3}$

We note that, the structure of the refined integer invariants (4.7) for $Q=1$ and $Q=5$ has the form (3.8) consistent with a contribution of genus- $g$ curves. For example, for $Q=1$ we have

$$
\sum_{s, r} D_{Q, s, r} q^{s} t^{r}=t\left[-\left(q^{-1} t^{-1 / 2}+q t^{1 / 2}\right)^{2}+2\right]
$$

which corresponds to a contribution of curves with $g=0$ and $g=1$. This is a strong evidence suggesting that embedding the knot homology in our physical setup is indeed correct. Moreover, since both terms in (4.11) have an extra factor of $t$, it also suggests, though not conclusively, that these curves are isolated and that we indeed have an affine change of basis in comparing knot homology with BPS spectrum, as discussed in the previous section. It would be extremely interesting to consider the Lagrangian D-brane associated to the trefoil and check whether or not there are isolated curves with $Q=1$ and $g=0,1$ ending on it.

We find similar contributions of genus- $g$ curves for other torus knots, $T_{2,2 m+1}$, where the maximal genus of such curves grows linearly with the number of crossings, $g_{\max }=m$. Again, these examples suggest that, in order to compare the invariants $D_{Q, s, r}$ with the

physical BPS degeneracies $\widetilde{D}_{Q, s, r}$, one has to make a change of basis, $r \rightarrow r-Q / 2$. Note that in the case of the unknot we did not have to change the basis. This suggests that the change of basis involves a simple knot invariant $\alpha_{K}, c f .(3.9)$.

\subsection{The Knot $5_{1}$}

Following the same reasoning as in the previous example, we consider the set of values $(Q, s)$ which correspond to non-zero integer invariants $N_{\square, Q, s}$ :

$$
(3,0), \quad(3,-4), \quad(3,4), \quad(5,0), \quad(5,-2), \quad(5,2), \quad(5,-4), \quad(5,4), \quad(7,-2),
$$

Again, following the natural assumption that these are the values of $(Q, s)$ which correspond to non-trivial elements in (1.7), we can find the invariants $D_{Q, s, r}$ which encode the $\operatorname{sl}(N)$ homological invariants,

$$
\begin{aligned}
& D_{5,-4,0}=1, \quad D_{5,-2,2}=1, \quad D_{7,-2,3}=1, \quad D_{5,2,4}=1, \quad D_{7,2,5}=1, \\
& D_{3,-4,0}=-1, \quad D_{3,0,2}=-1, \quad D_{5,0,3}=-1, \quad D_{3,4,4}=-1, \quad D_{5,4,5}=-1
\end{aligned}
$$


Given these numbers, one can use (1.8) to compute all $\operatorname{sl}(N)$ cohomology groups for the knot $5_{1}$. For example, in the case $N=3$ we get

$$
K h_{N=3}\left(5_{1}\right)=q^{6}+q^{8}+q^{10}+q^{10} t^{2}+q^{12} t^{2}+q^{16} t^{3}+q^{18} t^{3}+q^{14} t^{4}+q^{16} t^{4}+q^{20} t^{5}+q^{22} t^{5}
$$

Again, this result is in a perfect agreement with the $\operatorname{sl}(3)$ knot homology computed using the technique of foams [16]. It would be interesting to check the other $\operatorname{sl}(N)$ knot homology groups that follow from $(4.13)$.

It is easy to generalize these examples to arbitrary torus knots $T_{2,2 m+1}$. For every torus knot $T_{2,2 m+1}$, we find a complete agreement between our predictions based on (1.8) and the $\operatorname{sl}(3)$ knot homology groups computed using the technique of web cobordisms (the details will be discussed elsewhere [38]).

\section{Generalizations}

The proposed relation between the knot cohomology and the spectrum of BPS states leads to non-trivial predictions for both knot theory and physics. Apart from the computational predictions, examples of which we discussed in the previous section, there are interesting generalizations suggested by this relation. For example, it suggests that there should exist a categorification of more general knot invariants associated with arbitrary representations of $s l(N)$, not just the fundamental representation. Similarly, since knot cohomology can be defined over arbitrary ground fields, including finite number fields, there should exist corresponding physical realizations in terms of BPS states. This would be interesting to study further. Also, it would be very exciting to find a combinatorial definition of the integer invariants $D_{Q, s, r}$, and of their generalizations to other representations.

The formulation of the $s l(N)$ homological invariant in terms of the refined BPS invariants $D_{Q, s, r}$ can also be extended to links. Let $L$ be an oriented link in $\mathbf{S}^{3}$ with $\ell$ components, $K_{1}, \ldots, K_{\ell}$, all of which carry a fundamental representation of $s l(N)$. As in (1.1), the expectation value of the corresponding Wilson loop operator $W(L)=W_{\square, \cdots, \square}(L)$ is related to the polynomial $\operatorname{sl}(N)$ invariant, with a minor modification,

$$
P_{N}(L)=q^{-2 N \operatorname{lk}(L)}\langle W(L)\rangle
$$

where $\operatorname{lk}(L)=\sum_{a<b} \operatorname{lk}\left(K_{a}, K_{b}\right)$ is the total linking number of $L$. The formulation of $P_{N}(L)$ in terms of integer BPS invariants also needs some modification [8]. Namely, in our notations,

$$
\langle W(L)\rangle^{(c)}=\left(q^{-1}-q\right)^{\ell-2} \sum_{Q, s} N_{(\square, \cdots, \square), Q, s} q^{N Q+s}
$$


where $\langle W(L)\rangle^{(c)}$ is the connected correlation function. For example, for a two-component link, we have

$$
\langle W(L)\rangle^{(c)}=\langle W(L)\rangle-\left\langle W\left(K_{1}\right)\right\rangle\left\langle W\left(K_{2}\right)\right\rangle
$$

and

$$
P_{N}(L)=q^{-2 N \operatorname{lk}(L)}\left[P_{N}\left(K_{1}\right) P_{N}\left(K_{2}\right)+\sum_{Q, s} N_{(\square, \square), Q, s} q^{N Q+s}\right]
$$

where $P_{N}\left(K_{1}\right)$ and $P_{N}\left(K_{2}\right)$ denote the $s l(N)$ polynomials of the link components.

We wish to write a similar expression for the graded Poincaré polynomial $K h_{N}(L)$ in terms of integer invariants $D_{Q, s, r}(L)$. Because of the corrections involving the $\operatorname{sl}(N)$ invariants of the sublinks, this formulation is less obvious than in the case of knots. For example, for a two-component link $L$, we find, $c f$. (1.8),

$$
K h_{N}(L)=q^{-2 N \operatorname{lk}(L)}\left[t^{\alpha} K h_{N}\left(K_{1}\right) K h_{N}\left(K_{2}\right)+\frac{1}{q-q^{-1}} \sum_{Q, s, r \in \mathbb{Z}} D_{Q, s, r} q^{N Q+s} t^{r}\right]
$$

where $\alpha$ is a simple invariant of $L$, and the integer invariants $D_{Q, s, r}$ and $N_{(\square, \square), Q, s}$ are related as follows:

$$
N_{(\square, \square), Q, s-1}-N_{(\square, \square), Q, s+1}=\sum_{r \in \mathbb{Z}}(-1)^{r} D_{Q, s, r}
$$

Notice, this relation is quite different from what we had in the case of knots, $c f$. (1.9).

The first term in (5.5) is similar to the first term in (5.4). In order to understand the structure of the second term, note that the factor $\left(q^{-1}-q\right)^{\ell-2}$ in $(5.2)$ is a product

of two terms, $\left(q^{-1}-q\right)^{-1}$ and $\left(q^{-1}-q\right)^{\ell-1}$, which have different origin and should be treated differently. The first term, $\left(q^{-1}-q\right)^{-1}$, comes from the Schwinger computation [7] and remains intact once $P_{N}(L)$ is lifted to the homological $s l(N)$ invariant. On the other hand, the factor $\left(q^{-1}-q\right)^{\ell-1}$ is similar to the contribution of a genus- $g$ curve, $\left(q^{-1}-q\right)^{2 g}$, discussed in section 3. In the homological $\operatorname{sl}(N)$ invariant this factor is replaced by a polynomial expression in $q^{ \pm 1}$ and $t^{ \pm 1}$. Applying the same logic to (5.2) gives the second term in (5.5).

\section{Acknowledgments}

We would like to thank D. Bar-Natan, R. Dijkgraaf, M. Gross, K. Intriligator, A. Kapustin, M. Khovanov, A. Klemm, M. Mariño, H. Ooguri, J. Roberts and D. Thurston for 
useful discussions. S.G. would also like to thank the Caltech Particle Theory Group for kind hospitality. The work of A.S. is supported by NSF grant DMS-0204927. This work was conducted during the period S.G. served as a Clay Mathematics Institute Long-Term Prize Fellow. S.G. is also supported in part by RFBR grant 04-02-16880. The work of C.V. is supported in part by NSF grants PHY-0244821 and DMS-0244464.

Note added: A preliminary version of the present work was presented by one of us (S.G.) at Caltech and UCSD seminars in October 2004. Since then, there have been some interesting developments which provide additional support for the conjecture (1.8), see e.g. [39] for independent computations of the Khovanov-Rozansky homology in some of the examples considered here (see also [38]). 


\section{Appendix A. HOMFLY Polynomial for Some Knots}

Let us list the polynomial invariant $f_{\square}(q, \lambda)$ for some simple knots:

$$
\begin{aligned}
& f_{\square}\left(3_{1}\right)=\frac{\lambda q^{-2}+q^{2} \lambda-\lambda^{3}-\lambda^{3} q^{-2}-q^{2} \lambda^{3}+\lambda^{5}}{q^{-1}-q} \\
& f_{\square}\left(4_{1}\right)=\frac{\lambda^{-3}-\lambda^{-1} q^{-2}-q^{2} \lambda^{-1}+\lambda q^{-2}+\lambda q^{2}-\lambda^{3}}{q^{-1}-q} \\
& f_{\square}\left(5_{1}\right)=\frac{\lambda^{3}+\lambda^{3} q^{-4}+q^{4} \lambda^{3}-\lambda^{5}-\lambda^{5} q^{-4}-\lambda^{5} q^{-2}-q^{2} \lambda^{5}-q^{4} \lambda^{5}+\lambda^{7} q^{-2}+q^{2} \lambda^{7}}{q^{-1}-q} \\
& f_{\square}\left(6_{1}\right)=\frac{\lambda^{-3}+\lambda^{-1}-q^{-2} \lambda^{-1}-q^{2} \lambda^{-1}-\lambda+\lambda^{3} q^{-2}+q^{2} \lambda^{3}-\lambda^{5}}{q^{-1}-q}
\end{aligned}
$$

According to (2.6), the polynomial $f_{\square}(q, \lambda)$ can be viewed as a generating function of the BPS invariants $\widehat{N}_{\square, g, Q}$ (or $N_{\square, Q, s}$ ). Notice, that the BPS invariants for the knots $3_{1}, 4_{1}$, and $6_{1}$ obey a simple relation,

$$
\widehat{N}_{\square, g, Q}\left(3_{1}\right)-\widehat{N}_{\square, g, Q}\left(4_{1}\right)+\widehat{N}_{\square, g, Q}\left(6_{1}\right)=\widehat{N}_{\square, g, Q}(\text { unknot })
$$

\section{Appendix B. $s l(2)$ Knot Homology for Some Knots}

Here, following [18], we list Khovanov's $s l(2)$ invariants, $K h_{2}(L)$, for some simple knots:

$$
\begin{aligned}
& K h_{2}\left(3_{1}\right)=q+q^{3}+q^{5} t^{2}+q^{9} t^{3} \\
& K h_{2}\left(4_{1}\right)=q+q^{-1}+q^{-1} t^{-1}+q t+q^{-5} t^{-2}+q^{5} t^{2} \\
& K h_{2}\left(5_{1}\right)=q^{3}+q^{5}+q^{7} t^{2}+q^{11} t^{3}+q^{11} t^{4}+q^{15} t^{5} \\
& K h_{2}\left(5_{2}\right)=q+q^{3}+q^{3} t+q^{5} t^{2}+q^{7} t^{2}+q^{9} t^{3}+q^{9} t^{4}+q^{13} t^{5} \\
& K h_{2}\left(6_{1}\right)=2 q^{-1}+q^{-1} t^{-1}+q^{-5} t^{-2}+q+q t+q^{3} t+q^{5} t^{2}+q^{5} t^{3}+q^{9} t^{4} \\
& K h_{2}\left(7_{1}\right)=q^{5}+q^{7}+q^{9} t^{2}+q^{13} t^{3}+q^{13} t^{4}+q^{17} t^{5}+q^{17} t^{6}+q^{21} t^{7}
\end{aligned}
$$

Notice, that the $s l(2)$ homological invariants for the knots $3_{1}, 4_{1}$ and $6_{1}$ are closely related:

$$
\begin{aligned}
K h_{2}\left(3_{1}\right)-K h_{2}\left(4_{1}\right)+K h_{2}\left(6_{1}\right)= & q+q^{-1} \\
& +q^{3}(1+t)+q^{5}\left(t^{2}+t^{3}\right)+q^{9}\left(t^{3}+t^{4}\right)
\end{aligned}
$$

The right-hand side of this expression evaluated at $t=-1$ gives $K h_{2}$ (unknot), in agreement with (A.1). 


\section{References}

[1] A. Schwarz, "New topological invariants arising in the theory of quantized fields," Baku International Topological Conf., Abstracts (part II) (1987).

[2] E. Witten, "Quantum Field Theory And The Jones Polynomial," Commun. Math. Phys. 121, 351 (1989).

[3] P. Freyd, D. Yetter, J. Hoste, W. Lickorish, K. Millett, A. Oceanu, "A New Polynomial Invariant of Knots and Links," Bull. Amer. Math. Soc. 12 (1985) 239.

[4] E. Witten, "Chern-Simons gauge theory as a string theory," Prog. Math. 133 (1995) 637, hep-th/9207094.

[5] R. Gopakumar and C. Vafa, "On the gauge theory/geometry correspondence," Adv. Theor. Math. Phys. 3 (1999) 1415, hep-th/9811131.

[6] R. Gopakumar and C. Vafa, "M-theory and topological strings. I,II," hep-th/9809187; hep-th/9812127.

[7] H. Ooguri, C. Vafa, "Knot Invariants and Topological Strings," Nucl.Phys. B577 (2000) 419.

[8] J. M. F. Labastida, M. Marino and C. Vafa, "Knots, links and branes at large N," JHEP 0011, 007 (2000), hep-th/0010102.

[9] S. Hosono, M.-H. Saito, A. Takahashi, "Holomorphic Anomaly Equation and BPS State Counting of Rational Elliptic Surface," Adv.Theor.Math.Phys. 3 (1999) 177.

[10] S. Hosono, M.-H. Saito, A. Takahashi, "Relative Lefschetz Action and BPS State Counting," Internat. Math. Res. Notices, (2001), No. 15, 783.

[11] S. Katz, A. Klemm and C. Vafa, "M-theory, topological strings and spinning black holes," Adv. Theor. Math. Phys. 3 (1999) 1445, hep-th/9910181.

[12] D. Maulik, N. Nekrasov, A. Okounkov, R. Pandharipande, "Gromov-Witten theory and Donaldson-Thomas theory, I," math.AG/0312059.

[13] S. Katz, "Gromov-Witten, Gopakumar-Vafa, and Donaldson-Thomas invariants of Calabi-Yau threefolds," math.ag/0408266.

[14] M. Khovanov, "A categorification of the Jones polynomial," math.QA/9908171.

[15] M. Khovanov, "Categorifications of the colored Jones polynomial," math.QA/0302060.

[16] M. Khovanov, "sl(3) link homology I," math.QA/0304375.

[17] M. Khovanov, L. Rozansky, "Matrix factorizations and link homology," math.QA/0401268.

[18] D. Bar-Natan, "On Khovanov's categorification of the Jones polynomial," math.QA/0201043.

[19] M. Khovanov, "An invariant of tangle cobordisms," math.QA/0207264.

[20] M. Jacobsson, "An invariant of link cobordisms from Khovanov's homology theory," math.GT/0206303.

[21] A. Shumakovitch, KhoHo - a program for computing and studying Khovanov homology, http://www.geometrie.ch/KhoHo 
[22] T. J. Hollowood, A. Iqbal and C. Vafa, "Matrix models, geometric engineering and elliptic genera," hep-th/0310272.

[23] J. M. F. Labastida and M. Marino, "Polynomial invariants for torus knots and topological strings," Commun. Math. Phys. 217 (2001) 423, hep-th/0004196.

[24] J. M. F. Labastida and M. Marino, "A new point of view in the theory of knot and link invariants," math.qa/0104180.

[25] H. Murakami, T. Ohtsuki, S. Yamada, "HOMFLY polynomial via an invariant of colored plane graphs," Enseign. Math. 44 (1998) 325.

[26] P. S. Aspinwall, "D-branes on Calabi-Yau manifolds," hep-th/0403166.

[27] M. Kontsevich, unpublished.

[28] A. Kapustin and Y. Li, "D-branes in Landau-Ginzburg models and algebraic geometry," JHEP 0312 (2003) 005, hep-th/0210296.

[29] D. Orlov, "Triangulated Categories of Singularities and D-Branes in Landau-Ginzburg Orbifold," math.AG/0302304.

[30] I. Brunner, M. Herbst, W. Lerche and B. Scheuner, "Landau-Ginzburg realization of open string TFT," hep-th/0305133.

[31] S. K. Ashok, E. Dell'Aquila and D. E. Diaconescu, "Fractional branes in LandauGinzburg orbifolds," hep-th/0401135.

[32] S. K. Ashok, E. Dell'Aquila, D. E. Diaconescu and B. Florea, "Obstructed D-branes in Landau-Ginzburg orbifolds," hep-th/0404167.

[33] K. Hori and J. Walcher, "F-term equations near Gepner points," hep-th/0404196.

[34] I. Brunner, M. Herbst, W. Lerche and J. Walcher, "Matrix factorizations and mirror symmetry: The cubic curve," hep-th/0408243.

[35] "Mirror Symmetry" (Clay Mathematics Monographs, V. 1), K. Hori et.al. ed, American Mathematical Society, 2003.

[36] A. Schwarz and I. Shapiro, "Some remarks on Gopakumar-Vafa invariants," hepth/0412119.

[37] C. Taubes, "Lagrangians for the Gopakumar-Vafa conjecture," math.DG/0201219.

[38] N. Dunfield, S. Gukov, J. Rasmussen, "The Superpolynomial for Knot Homologies," math.GT/0505662.

[39] D. Bar-Natan, "Some Khovanov-Rozansky Computations" ; http://www.math.toronto.edu/drorbn/Misc/KhovanovRozansky/index.html 